\title{
BROWNIAN MOTIONS OF ELLIPSOIDS
}

\author{
BY
}

\author{
J. R. NORRIS, L. C. G. ROGERS AND DAVID WILLIAMS
}

\begin{abstract}
The object of this paper is to provide an elementary treatment (involving no differential geometry) of Brownian motions of ellipsoids, and, in particular, of some remarkable results first obtained by Dynkin.

The canonical right-invariant Brownian motion $G=\{G(t)\}$ on $\mathrm{GL}(n)$ induces processes $X=G G^{T}$ and $Y=G^{T} G$ on the space of positive-definite symmetric matrices. The motion of the common eigenvalues of $X$ and $Y$ is analysed. It is further shown that the orthonormal frame of eigenvectors of $X$ ultimately behaves like Brownian motion on $\mathrm{O}(n)$, while that of $Y$ converges to a limiting value.

The $Y$ process is that studied by Dynkin and Orihara. From a naive standpoint, the $X$ process would seem to provide a more natural model.
\end{abstract}

1. The object of this note is to present a strictly elementary account of Brownian motions of ellipsoids.

Throughout, we use $d$ to signify the Itô differential, and $\partial$ to signify the Stratonovich differential. Recall that if $x$ and $y$ are continuous semimartingales, then

$$
x \partial y=x d y+\frac{1}{2} d x d y
$$

where $d x d y=d[x, y]$. See, for example, Chapter III of Ikeda and Watanabe [3]. Relation (1.1) extends to matrix-valued semimartingales with the obvious componentwise interpretation.

2. Let $B$ be a process taking values in the set of all $n \times n$ matrices such that the components of $B$ are independent Brownian motions on $\mathbf{R}$. We construct a right-invariant Brownian motion $G$ on the group $\mathrm{GL}(n)$ of invertible $n \times n$ matrices by solving

$$
\partial G=(\partial B) G, \quad G(0)=I,
$$

where $I$ is the identity $n \times n$ matrix. On taking the Stratonovich differential of the equation $G G^{-1}=I$, we find that

$$
\partial\left(G^{-1}\right)=-G^{-1} \partial B .
$$

The statement that $G$ is a right-invariant Brownian motion means that for each $u>0$, the process $\left\{G(t+u) G(u)^{-1}: t \geq 0\right\}$ is identical in law to $G$ and is independent of the process $\{G(r): r \leq u\}$. Note that since

$$
\partial\left(G^{-1}\right)^{T}=-\left(\partial B^{T}\right)\left(G^{-1}\right)^{T}
$$

Received by the editors May 23, 1985 .

1980 Mathematics Subject Classification. Primary 60J60, 60J65, 58G32; Secondary 15A52, $53 \mathrm{C} 35,60 \mathrm{~J} 30$.

Key words and phrases. Brownian motions of ellipsoids, eigenvalues, eigenvectors, positivedefinite symmetric matrices, orthogonal group, Stratonovich differential, Itô's formula. 
the superscript $T$ signifying transpose, it follows that

$$
\left(G^{-1}\right)^{T} \text { has the same law as } G .
$$

We shall write $S$ and $A$ for the symmetric and antisymmetric parts of the driving Brownian motion $B$

$$
S=\frac{1}{2}\left(B+B^{T}\right), \quad A=\frac{1}{2}\left(B-B^{T}\right) .
$$

3. If $v(0)$ is a fixed vector in $\mathbf{R}^{n}$ and we set $v(t)=G(t) v(0)$, then

$$
\partial v(t)=(\partial B) v(t)
$$

so that $v=\{v(t)\}$ satisfies an autonomous stochastic differential equation. Thus $v$ is a Markov process which is easily shown to have generator $\frac{1}{2}|v|^{2} \Delta+\frac{1}{2} v \cdot \operatorname{grad}$. The process $G$ is therefore the flow associated with equation (3.1). Under the flow $G$, the points which at time 0 lie on the unit sphere will at time $t$ lie on the ellipsoid $v^{T} Z(t) v=1$, where $Z(t)=\left(G^{-1}\right)^{T} G^{-1}$. Because $G$ and $\left(G^{-1}\right)^{T}$ are identical in law, we shall for notational simplicity study the process

$$
X=G G^{T} \text {. }
$$

Since

$$
\partial X=(\partial B) X+X\left(\partial B^{T}\right)
$$

$X$ is clearly a Markov process on the space of positive-definite symmetric matrices, or, equivalently, on the space of ellipsoids.

4. Define the 'companion' process

$$
Y=G^{T} G .
$$

We have

$$
\partial Y=G^{T}\left(\partial B^{T}+\partial B\right) G=2 G^{T}(\partial S) G,
$$

and though this is not autonomous in $Y$, it is even so true that

$Y$ is a Markov process on the space of ellipsoids.

We prove this below. The process $Y$ has no direct 'flow' interpretation. However,

$$
\begin{aligned}
& \text { the law of } Y \text { is invariant under transformations } Y \rightarrow K^{T} Y K \text {, } \\
& K \text { being a fixed element of } \operatorname{GL}(n) \text {. }
\end{aligned}
$$

This property is regarded as fundamental in the 'symmetric space' approach in Dynkin's remarkable paper $[\mathbf{1}]$ and in the fine subsequent work [5] by Orihara. The simple construction of $Y$ afforded by equation (4.1) allows us to give rapid proofs of many of Dynkin's results.

From our point of view, the process $X$ is more natural. Its asymptotic behaviour is quite different from that of $Y$. 
5. It simplified the earlier exposition to assume that $G(0)=I$. However, it is now convenient to suppose that $G(0)$ is chosen so that $X(0)$ has distinct eigenvalues. Otherwise, there will be 'infinite spinning' of eigenvectors within any time interval $(0, \varepsilon)$. Of course for every $t, X(t)$ and $Y(t)$ have the same set of eigenvalues. We shall prove that, with probability 1 ,

$$
X(t) \text { has distinct eigenvalues for every } t \text {. }
$$

We label the eigenvalues $\lambda_{i}(t)$ of $X(t)$ so that

$$
0<\lambda_{1}(t)<\lambda_{2}(t)<\cdots<\lambda_{n}(t)
$$

and write $\Lambda=\{\Lambda(t)\}$ for the process

$$
\Lambda=\operatorname{diag}\left(\lambda_{i}\right) .
$$

It will be convenient to write

$$
\gamma_{i}(t)=\frac{1}{2} \log \lambda_{i}(t), \quad \Gamma=\operatorname{diag}\left(\gamma_{i}\right) .
$$

If result (5.1) is assumed, it is standard that there exist continuous semimartingales $P$ and $Q$ with values in the group $\mathrm{O}(n)$ of orthogonal $n \times n$ matrices such that

$$
P^{T} X P=Q^{T} Y Q=\Lambda .
$$

We shall need the fact that (at each time $t$ )

$$
\Phi=P^{T} G Q \text { is diagonal and } \Phi^{2}=\Lambda .
$$

Proof OF (5.6). From (5.5) and the definitions of $X$ and $Y, \Phi \Phi^{T}=\Phi^{T} \Phi=\Lambda$, so that $H_{1}=\Lambda^{-1 / 2} \Phi$ and $H_{2}=\Phi \Lambda^{-1 / 2}$ are orthogonal. Now, $\Phi=\Lambda^{1 / 2} H_{1}=$ $H_{2} \Lambda^{1 / 2}$. The $n$th row of $\Lambda^{1 / 2} H_{1}$ has norm $\lambda_{n}^{1 / 2}$. But the $n$th row of $H_{2} \Lambda^{1 / 2}$ can have norm $\lambda_{n}^{1 / 2}$ only if its diagonal entry is its only nonzero entry. By proceeding inductively, one shows that $\Phi$ is diagonal.

We can suppose that $P(0)$ and $Q(0)$ are chosen so that $\Phi_{i i}(0)>0$ for each $i$. Then, because of continuity,

$$
\Phi=\Lambda^{1 / 2}
$$

for all $t$.

On differentiating $P P^{T}=I$, one sees that processes $M$ and $N$ defined via

$$
\partial M=P^{-1} \partial P, \quad \partial N=Q^{-1} \partial Q, \quad M(0)=N(0)=0,
$$

take values in the space of skew-symmetric matrices (the Lie algebra of $\mathrm{O}(n)$ ).

6. We collect together various equations:

$$
\begin{aligned}
& \partial G=(\partial B) G=(\partial S+\partial A) G, \\
& X=G G^{T}, \quad Y=G^{T} G, \quad \partial X=(\partial B) X+X(\partial B)^{T}, \\
& P^{T} X P=Q^{T} Y Q=\Lambda, \\
& \partial P=P \partial M, \quad \partial Q=Q \partial N, \quad M=-M^{T}, \quad N=-N^{T}, \\
& P^{T} G Q=\Lambda^{1 / 2}, \\
& \Gamma=\frac{1}{2} \log \Lambda .
\end{aligned}
$$


We now develop stochastic differential equations valid up to the time $\zeta$ of the first collision of eigenvalues of $X$ (later to be proved to be infinite). We concentrate on the process $X$.

Since $\Lambda=P^{T} X P$ and $\partial P=P \partial M$, we have

$$
\begin{aligned}
\partial \Lambda & =\left(\partial M^{T}\right) \Lambda+\Lambda(\partial M)+P^{T}\left[(\partial B) X+X\left(\partial B^{T}\right)\right] P \\
& =\left(\partial M^{T}\right) \Lambda+\Lambda(\partial M)+(\partial \tilde{B}) \Lambda+\Lambda\left(\partial \tilde{B}^{T}\right),
\end{aligned}
$$

where the important process $\tilde{B}$ is defined via

$$
\partial \tilde{B}=P^{T}(\partial B) P .
$$

Because $P$ is orthogonal, it is easy to deduce the result

$$
\left(d \tilde{B}_{i j}\right)\left(d \tilde{B}_{k l}\right)= \begin{cases}d t & \text { if }(i, j)=(k, l) \\ 0 & \text { otherwise }\end{cases}
$$

from the corresponding result for $B$. Let $\tilde{S}$ and $\tilde{A}$ denote the symmetric and antisymmetric parts of $\tilde{B}$, and note that

$$
\partial \tilde{S}=P^{T}(\partial S) P, \quad \partial \tilde{A}=P^{T}(\partial A) P .
$$

If we make a $2 n^{2} \times 2 n^{2}$ multiplication table for the $2 n^{2}$ 'stochastic 1-forms' amongst the $d \tilde{S}_{i j}$ and $d \tilde{A}_{k l}$, it follows from (6.9) that all products will be 0 except for the cases

$$
\begin{aligned}
& \left(d \tilde{S}_{i i}\right)^{2}=d t, \quad\left(d \tilde{S}_{i j}\right)^{2}=\left(d \tilde{S}_{i j}\right)\left(d \tilde{S}_{j i}\right)=\frac{1}{2} d t \quad(i \neq j), \\
& \left(d \tilde{A}_{i j}\right)^{2}=-\left(d \tilde{A}_{i j}\right)\left(d \tilde{A}_{j i}\right)=\frac{1}{2} d t \quad(i \neq j) .
\end{aligned}
$$

From (6.7) we have, after some rearranging,

$$
\begin{aligned}
& \partial M_{i j}=\partial \tilde{A}_{i j}+\left(\lambda_{j}-\lambda_{i}\right)^{-1}\left(\lambda_{j}+\lambda_{i}\right) \partial \tilde{S}_{i j} \quad(i \neq j), \\
& \partial \lambda_{i}=2 \lambda_{i} \partial \tilde{B}_{i i} .
\end{aligned}
$$

Note that since $\gamma_{i}=\frac{1}{2} \log \lambda_{i}$, we may rewrite equations (6.13) and (6.14) as

$$
\begin{aligned}
& \partial M_{i j}=\partial \tilde{A}_{i j}+\operatorname{coth}\left(\gamma_{j}-\gamma_{i}\right) \partial \tilde{S}_{i j} \quad(i \neq j), \\
& \partial \gamma_{i}=\partial \tilde{B}_{i i} .
\end{aligned}
$$
$X$.

7. We now derive an autonomous Itô equation for the family of eigenvalues of

From (6.8) we obtain

$$
d \tilde{B}=P^{T}(d B) P+\frac{1}{2}\left(d M^{T}\right)(d \tilde{B})+\frac{1}{2}(d \tilde{B})(d M)=d W+d F .
$$

Here, $d W=P^{T}(d B) P$, so that $W$ has independent Brownian components because of Lévy's theorem and the results at (6.9), and $F$ is a process of finite variation with

$$
d F_{i j}=\sum_{p} \frac{1}{2}\left(d M_{p i}\right)\left(d \tilde{B}_{p j}\right)+\sum_{p} \frac{1}{2}\left(d \tilde{B}_{i p}\right)\left(d M_{p j}\right) .
$$

From (6.13) and (6.9), it is immediate that

$$
\begin{aligned}
& d F_{i j}=0 \quad(i \neq j), \\
& d F_{i i}=\sum_{p}\left(d M_{p i}\right)\left(d \tilde{S}_{p i}\right)=\frac{1}{2} \sum_{p \neq i} \operatorname{coth}\left(\gamma_{i}-\gamma_{p}\right) d t .
\end{aligned}
$$


Thus

$$
d \gamma_{i}=d \tilde{B}_{i i}=d W_{i i}+\frac{1}{2} \sum_{p \neq i} \operatorname{coth}\left(\gamma_{i}-\gamma_{p}\right) d t
$$

Here is the complete description of the eigenvalue motion.

THEOREM A. The eigenvalues of $X$ never collide. Label them as $\left(\lambda_{i}\right)$, where $0<\lambda_{1}<\lambda_{2}<\cdots<\lambda_{n}$. Let $\gamma_{i}=\frac{1}{2} \log \lambda_{i}$. Then $\left(\gamma_{i}: 1 \leq i \leq n\right)$ satisfies an Itô equation

$$
d \gamma_{i}=d \beta_{i}+\frac{1}{2} \sum_{p \neq i} \operatorname{coth}\left(\gamma_{i}-\gamma_{p}\right) d t
$$

where $\left(\beta_{i}: 1 \leq i \leq n\right)$ are independent Brownian motions. Moreover,

$$
\lim _{t \uparrow \infty} t^{-1} \log \lambda_{i}(t)=2 i-(n+1) .
$$

We defer the proof of this Theorem until $\S \S 11-12$ (though you can read it now if you wish) so that we can see what consequences the theorem has for the motion of the eigenvectors of $X$ and those of $Y$.

8. We see from equation (6.14) that $\lambda_{i}$ obtains its quadratic variation from that of the diagonal element $\tilde{B}_{i i}$ of $\tilde{B}$. Because there is no quadratic covariation between different components of $\tilde{B}$ (see (6.9)) we see that in transforming (6.13) from its Stratonovich to its Itô form, there are no 'correction' terms. Thus

$$
d M_{i j}=d \tilde{A}_{i j}+\left(\lambda_{j}-\lambda_{i}\right)^{-1}\left(\lambda_{j}+\lambda_{i}\right) d \tilde{S}_{i j} \quad(i \neq j),
$$

and by (7.2) we see that $M_{i j}$ is a local martingale.

The important point about the eigenvector motion of $X$ is the following. Result (7.4) implies that for $i<j$,

$$
\left(\lambda_{j}-\lambda_{i}\right)^{-1}\left(\lambda_{j}+\lambda_{i}\right) \rightarrow 1 \quad(t \rightarrow \infty)
$$

It is now clear from (8.1) that in the long run, the orthonormal frame $P$ of eigenvectors of $X$ behaves very much like the solution $\tilde{P}$ of $\partial \tilde{P}=\tilde{P} \partial \tilde{L}$, where $\tilde{L}$ is a process on the set of skew-symmetric matrices such that $\left(\tilde{L}_{i j}: i<j\right)$ are independent Brownian motions (as are the $\left(\tilde{B}_{i j}: i<j\right)$ ). The motion $\tilde{P}$ is canonical (2-sided-invariant) Brownian motion on $\mathrm{O}(n)$. Thus,

$$
\begin{aligned}
& \text { ultimately, the behaviour of the orthonormal frame } P \text { of eigenvectors } \\
& \text { of } X \text { is just like Brownian motion on } \mathrm{O}(n) \text {. }
\end{aligned}
$$

9. The eigenvectors of $Y$ behave very differently! (You may wish to refresh your memory of $Y$ by rereading equations (6.1)-(6.6).)

From (6.5) and (6.3), $G=P \Lambda^{1 / 2} Q^{T}=P Q^{T} Y^{1 / 2}$. Hence

$$
\begin{aligned}
\partial Y & =G^{T}\left(\partial B^{T}+\partial B\right) G=2 Y^{1 / 2} Q P^{T}(\partial S) P Q^{T} Y^{1 / 2} \\
& =2 Y^{1 / 2} Q(\partial \tilde{S}) Q^{T} Y^{1 / 2}
\end{aligned}
$$

Next,

$$
\begin{aligned}
\partial \Lambda & =\partial\left(Q^{T} Y Q\right)=\left(\partial N^{T}\right) \Lambda+\Lambda(\partial N)+Q^{T}(\partial Y) Q \\
& =\left(\partial N^{T}\right) \Lambda+\Lambda(\partial N)+2 \Lambda^{1 / 2}(\partial \tilde{S}) \Lambda^{1 / 2}
\end{aligned}
$$


This yields the equation

$$
\partial N_{i j}=\left(\lambda_{j}-\lambda_{i}\right)^{-1} 2 \lambda_{i}^{1 / 2} \lambda_{j}^{1 / 2} \partial \tilde{S}_{i j} \quad(i \neq j) .
$$

The reasoning leading to equation (8.1) shows that we have the corresponding Itô equation:

$$
d N_{i j}=\left(\lambda_{j}-\lambda_{i}\right)^{-1} 2 \lambda_{i}^{1 / 2} \lambda_{j}^{1 / 2} d \tilde{S}_{i j} \quad(i \neq j),
$$

and hence, using result (7.4), we find that

$$
\left[N_{i j}\right](\infty)=2 \int_{0}^{\infty}\left\{\left(\lambda_{j}-\lambda_{i}\right)^{-2} \lambda_{i} \lambda_{j}\right\}(s) d s<\infty
$$

The equation $\partial Q=Q \partial N$ has Itô form $d Q=Q d N+\frac{1}{2} Q d[N]$. Since $\left|Q_{k l}\right| \leq 1$, $\forall(k, l)$, it follows from (9.4) that for every pair $(i, j)$,

$$
\begin{aligned}
& \text { the local martingale part of } Q_{i j} \text { has finite total quadratic } \\
& \text { variation }\left[Q_{i j}\right](\infty)
\end{aligned}
$$

and

(9.6) the finite-variation part of $Q_{i j}$ has finite total variation on $[0, \infty)$.

The following theorem is an immediate consequence.

THEOREM B. With probability 1 , the orthonormal frame $\{Q(t)\}$ of eigenvectors of $\{Y(t)\}$ has the property that $Q_{i j}(\infty)=\lim _{t \uparrow \infty} Q_{i j}(t)$ exists.

10. We have not yet established the Markov property of $Y$. Let us do so.

Put $\partial \hat{S}=Q^{T}(\partial \tilde{S}) Q$. Since $Q$ is orthogonal, it is easily checked that (compare (6.11))

$$
d \hat{S}_{p r} d \hat{S}_{u v}=\frac{1}{2}\left[I_{p u} I_{r v}+I_{p v} I_{r u}\right] d t
$$

and we then find from equation (9.1) that

$$
d Y_{i j} d Y_{k l}=2\left[Y_{i k} Y_{j l}+Y_{i l} Y_{j k}\right] d t .
$$

Note that, since $\partial G=(\partial B) G$, then

$$
d G=(d B) G+\frac{1}{2}(d B)^{2} G=(d B) G+\frac{1}{2} G d t .
$$

Hence, if $Y^{f}$ denotes the finite-variation part of $Y$, then

$$
\begin{aligned}
d Y^{f} & =d\left(G^{T} G\right)^{f}=\frac{1}{2} G^{T} G d t+\frac{1}{2} G^{T} G d t+\left(d G^{T}\right)(d G) \\
& =Y d t+G^{T}\left(d B^{T}\right)(d B) G=(n+1) Y d t .
\end{aligned}
$$

It follows from (10.1) and (10.2) that $Y$ is a Markov process. We regard $Y$ as parametrized by $\left\{Y_{i j}: i \leq j\right\}$. We see that the generator of $Y$ is given by

$$
g^{Y}=\sum_{i \leq j} \sum_{k \leq l} \sum_{k}\left(y_{i k} y_{j l}+y_{i l} y_{j k}\right) D_{i j} D_{k l}+(n+1) \sum_{i \leq j} \sum_{j} y_{i j} D_{i j},
$$

where $D_{i j}=\partial / \partial y_{i j}$ and $y_{j i}=y_{i j}$.

Dynkin obtained Theorem B-or, more precisely, an analogue of it (see $\S 13$ below)-by studying the potential theory associated with $\mathcal{G}^{Y}$. An immediate consequence of Theorem $B$ is that $\mathcal{G}^{Y}$ has a rich family of positive harmonic functions. See Dynkin's paper for a deep study. 
11. We now prove that the eigenvalues never collide.

The proof requires the following lemma.

LEMmA C. Suppose that $\lambda_{1}, \lambda_{2}, \ldots, \lambda_{n}$ are distinct, and let

$$
\sigma=\sum_{i} \sum_{j} \sum_{k} \frac{\lambda_{i}+\lambda_{j}}{\lambda_{i}-\lambda_{j}} \frac{\lambda_{i}+\lambda_{k}}{\lambda_{i}-\lambda_{k}}
$$

Then $\sigma=n(n-1)(n-2) / 3$.

PROOF. Obtain $3 \sigma$ by permuting $i, j$ and $k$ cyclically within the summation.

Recall that if $\gamma_{i}=\frac{1}{2} \log \lambda_{i}$, then

$$
d \gamma_{i}=d \beta_{i}+\frac{1}{2} \sum_{p \neq i} \operatorname{coth}\left(\gamma_{i}-\gamma_{p}\right) d t
$$

Define a 'potential function' $\theta$ by setting

$$
\theta(\gamma)=-\sum_{i>j} \log \sinh \left(\gamma_{i}-\gamma_{j}\right)
$$

Then equation (11.1) reads

$$
d \gamma_{i}=d \beta_{i}-\frac{1}{2} D_{i} \theta(\gamma) d t, \quad D_{i}=\partial / \partial \gamma_{i},
$$

and Itô's formula gives the equation

$$
d \theta(\gamma)=\sum_{i} D_{i} \theta(\gamma) d \beta_{i}+\frac{1}{2} \alpha(\gamma) d t
$$

where $\alpha(\gamma)=-\sum_{i}\left[D_{i} \theta(\gamma)\right]^{2}+\Delta \theta(\gamma)$. But, because

$$
\operatorname{coth}\left(\gamma_{i}-\gamma_{p}\right)=\left(\lambda_{i}+\lambda_{p}\right) /\left(\lambda_{i}-\lambda_{p}\right)
$$

we can use Lemma $\mathrm{C}$ to show that $\alpha(\gamma)=-(n+1) n(n-1) / 3$. Hence, by equation (11.4),

$$
\rho(\gamma)=\theta(\gamma)+(n+1) n(n-1) t / 6
$$

defines a continuous local martingale $\rho$ up to the explosion time $\zeta$ of $\rho$. Since $\rho$ is therefore a time-transformation of Brownian motion, it follows that if $\zeta<\infty$, then both of the following equalities hold:

$$
\limsup _{t \uparrow \varsigma} \rho(t)=\infty, \quad \liminf _{t \uparrow \varsigma} \rho(t)=-\infty .
$$

But the latter possibility cannot occur because the eigenvalues are bounded on compact intervals. Hence $s=\infty$ (with probability 1 ), and the eigenvalues never collide.

12. To finish the proof of Theorem A, we must prove the result (7.4), or equivalently that

$$
\lim _{t \uparrow \infty} t^{-1} \gamma_{i}(t)=c_{i}, \quad \text { where } c_{i}=i-\frac{1}{2}(n+1) .
$$

We may rewrite equation (7.3) as

$$
d \gamma_{i}=d \beta_{i}+c_{i} d t+\left\{\sum_{p<i} \lambda_{p}\left(\lambda_{i}-\lambda_{p}\right)^{-1}+\sum_{p>i} \lambda_{i}\left(\lambda_{i}-\lambda_{p}\right)^{-1}\right\} d t
$$


It is therefore immediate to check that if we write

$$
\varepsilon_{i}=\gamma_{i+1}-\gamma_{i}
$$

then $d \varepsilon_{i}=d \beta_{i+1}-d \beta_{i}+d t+b_{i}(t) d t$, where

$$
b_{i}<2 \lambda_{i}\left(\lambda_{i+1}-\lambda_{i}\right)^{-1}=2\left[\exp \left(2 \varepsilon_{i}\right)-1\right]^{-1} \text {. }
$$

We have

$$
\varepsilon_{i}(t)=\varepsilon_{i}(0)+\beta_{i+1}(t)-\beta_{i}(t)+t+\int_{0}^{t} b_{i}(s) d s .
$$

For comparison purposes, introduce the solution $\mu_{i}$ of the equation

$$
\mu_{i}(t)=\varepsilon_{i}(0)+\beta_{i+1}(t)-\beta_{i}(t)+t+\int_{0}^{t} 2\left[\exp \left(2 \mu_{i}(s)\right)-1\right]^{-1} d s .
$$

Let $\theta_{i}=\mu_{i}-\varepsilon_{i}$. Then

$$
\theta_{i}(t)=\int_{0}^{t} 2\left[\exp \left(2 \mu_{i}(s)\right)-1\right]^{-1} d s-\int_{0}^{t} b_{i}(s) d s,
$$

and $\theta_{i}$ has a continuous derivative. Let

$$
T_{i}=\inf \left\{t: \theta_{i}(t)<0\right\} \text {. }
$$

Then, using (12.3), we see that $T_{i}<\infty$ implies that $\varepsilon_{i}\left(T_{i}\right)=\mu_{i}\left(T_{i}\right)$ and $\theta_{i}^{\prime}\left(T_{i}\right)>0$, which is absurd. Hence $\theta_{i}(t) \geq 0, \forall t$, and $\varepsilon_{i}(t) \leq \mu_{i}(t)$. But is is easy to deduce from equation (12.4) that $\lim t^{-1} \mu_{i}(t)=1$, so that

$$
\limsup t^{-1}\left\{\gamma_{i+1}(t)-\gamma_{i}(t)\right\}=\lim \sup t^{-1} \varepsilon_{i}(t) \leq 1 .
$$

However, it is obvious from equation (7.3) that

$$
\limsup t^{-1} \gamma_{1}(t) \leq \frac{1}{2}(1-n), \quad \liminf t^{-1} \gamma_{n}(t) \geq \frac{1}{2}(n-1) .
$$

The only way in which results (12.5) and (12.6) can be compatible is for result (12.1) to hold.

All of the results announced up to this stage of the paper are now proved.

13. Brownian motions of ellipsoids of unit volume. What Dynkin actually studied was the process analogous to $Y$ on the space of ellipsoids of unit volume. Our results transfer to Brownian motions of ellipsoids of unit volume in a simple and natural way. We now list the main modifications which are necessary.

We replace the basic driving Brownian motion $B$ by

$$
B^{\text {new }}=B-n^{-1} \operatorname{Tr}(B) I,
$$

where $\operatorname{Tr}(B)=\sum_{i} B_{i i}$, the trace of $B$. Then $B^{\text {new }}$ has trace 0 . We introduce analogues of $G, X$ and $Y$ via

$$
\begin{array}{ll}
\partial G^{\text {new }}=\left(\partial B^{\text {new }}\right) G^{\text {new }}, & G^{\text {new }}(0)=I, \\
X^{\text {new }}=\left(G^{\text {new }}\right)\left(G^{\text {new }}\right)^{T}, \quad Y^{\text {new }}=\left(G^{\text {new }}\right)^{T}\left(G^{\text {new }}\right) .
\end{array}
$$

Then $G^{\text {new }}$ is a right-invariant Brownian motion on the group $\operatorname{SL}(n)$ of $n \times n$ matrices of determinant 1 . The process $Y^{\text {new }}$ has the invariance property analogous to property (4.4) in which $K$ is restricted to belong to $\operatorname{SL}(n)$. The analogue of 
Theorem $\mathrm{A}$ in $\S 7$ holds for the eigenvalues of $X^{\text {new }}$ and $Y^{\text {new }}$ provided that we replace $\beta$ in equation (7.3) by $\beta^{\text {new }}$, where

$$
\beta_{i}^{\text {new }}=\beta_{i}-n^{-1} \sum \beta_{k},
$$

so that $\beta^{\text {new }}$ is a Brownian motion on $\mathbf{R}^{n}$ conditioned so that $\sum_{i} \beta_{i}^{\text {new }}=0$. The eigenvectors of $X^{\text {new }}$ satisfy the analogue of result (8.2), and those of $Y^{\text {new }}$ the analogue of Theorem B in $\S 9$. The generator $\mathcal{G}_{\text {new }}^{Y}$ of $Y^{\text {new }}$ is obtained from the generator $g^{Y}$ of $Y$ at equation (10.3) as follows:

$$
\mathcal{G}_{\text {new }}^{Y}=\mathcal{G}^{Y}-2 n^{-1} \sum_{i \leq j} \sum_{k \leq l} \sum_{i j} y_{i j} y_{k l} D_{i j} D_{k l}
$$

14. The processes on symmetric matrices studied by Dyson [2] and McKean [4] may be handled in a similar way by exploiting the 'skew-product' representation into autonomous eigenvalue motion and eigenvector motion for which the natural clock depends on the eigenvalue process. This was done by one of us (D.W.) in an unpublished note.

15. Concluding remarks. We came to write this paper because, having very little knowledge of differential geometry, we cannot understand the papers of Dynkin and Orihara. We therefore set ourselves two tasks: first, to try to formulate and to prove the results directly; and second, to try to learn the differential geometry. This paper represents part of our efforts at the first task, and we hope it will be of interest even to those who do know the geometry. We hope to extend the naive approach into other areas in a subsequent paper. We look forward to our second task, aware that it will be more difficult, but convinced by the splendid results obtained by Dynkin and Orihara that it will be worthwhile.

\section{REFERENCES}

1. E. B. Dynkin, Non-negative eigenfunctions of the Laplace-Beltrami operator and Brownian motion in certain symmetric spaces, Dokl. Akad. Nauk SSSR 141 (1961), 1433-1436.

2. F. J. Dyson, A Brownian-motion model for the eigenvalues of a random matrix, J. Math. Phys. 3 (1962), 1191-1198.

3. N. Ikeda and S. Watanabe, Stochastic differential equations and diffusion processes, North-Holland, Amsterdam, 1981.

4. H. P. McKean, Stochastic integrals, Academic Press, New York and London, 1969.

5. A. Orihara, On random ellipsoid, J. Fac. Sci. Univ. Tokyo Sect. IA Math. 17 (1970), 73-85.

DePARTMENT OF MATHEMATICS AND COMPUTER SCIENCE, UNiversity College of Swansea, Singleton Park, Swansea, SA2 8PP, Great Britain

Current address: Statistical Laboratory, Department of Pure Mathematics and Mathematical Statistics, University of Cambridge, 16 Mill Lane, Cambridge CB2 1SB, Great Britain 